\title{
Context-Sensitive Control of Adaptation: Self-Modeling Networks for Human Mental Processes Using Mental Models
}

\author{
Raj BHALWANKAR ${ }^{\mathrm{a}}$, Laila VAN MENTS ${ }^{\mathrm{b}}$ and Jan TREUR ${ }^{\mathrm{a}, 1}$ \\ ${ }^{a}$ Social AI Group, Vrije Universiteit Amsterdam, Amsterdam, The Netherlands \\ ${ }^{\mathrm{b}}$ AutoLeadStar, Jerusalem, Israel
}

\begin{abstract}
Within their mental and social processes, humans often learn, adapt and apply specific mental models of processes in the world or other persons, as a kind of blueprints. In this paper, it is discussed how analysis of this provides useful inspiration for the development of new computational approaches from a Machine Learning and Network-Oriented Modeling perspective. Three main elements are: applying the mental model by internal simulation, developing and revising a mental model by some form of adaptation, and exerting control over this adaptation in a context-sensitive manner. This concept of controlled adaptation relates to the Plasticity Versus Stability Conundrum from neuroscience. The presented analysis has led to a three-level computational architecture for controlled adaptation. It is discussed and illustrated by examples of applications how this three-level computational architecture can be specified based on a self-modeling network and used to model controlled learning and adaptation processes based on mental models in a context-sensitive manner.
\end{abstract}

Keywords. Mental model, Adaptive network, Control of adaptation, Self-modeling network

\section{Introduction}

Historically, analysis of human neural, mental and social processes often has provided inspiration for interesting new methods and techniques in AI and Machine Learning. In this paper, the focus is on how in their mental and social processes, humans often learn, adapt and apply specific mental models as a kind of blueprints, schemas or maps. Although a vast majority of the large amounts of publications on mental models in multiple disciplines is informal and not computational, it is shown how analysis of them still can be used as inspiration for the development of new computational approaches from an AI and Machine Learning perspective. Three elements came out of this analysis: (1) applying a mental model by internal (mental) simulation, (2) to develop and maintain a mental model, adaptation of it takes place (which usually involves learning, extinction or forgetting, and revision), and (3) in a context-sensitive manner, control is exerted over this adaptation. Here in particular (3) is an interesting topic. In a wider neuroscientific context, for example, in [1], this topic is discussed in relation to what is called the Plasticity Versus Stability Conundrum and to the notion of metaplasticity; e.g., [2]. It has

${ }^{1}$ Corresponding Author: Jan Treur, Social AI Group, Vrije Universiteit Amsterdam, De Boelelaan 1111, 1081HV Amsterdam, the Netherlands; Email: j.treur@vu.nl. 
been found that in the brain plasticity is not constant and various neural mechanisms have been discovered by which the extent of plasticity varies by being controlled in a context-sensitive manner; $[1,2,3,4]$. Also, within an AI-context, in machine learning examples of this conundrum and controlled adaptation to address it are known, such as the (decreasing) temperature parameter in simulated annealing and the sensitive balancing between exploration and exploitation in reinforcement learning, also called the explore-exploit dilemma [5, 6, 7]. This is explained in [7] as follows.

'When you go to your favourite restaurant, do you always order the same thing, or do you try something new? Sticking with an old favourite ensures a good meal, but if you are willing to explore you might discover something better. This simple conundrum, deciding between something you know and something you do not, is commonly referred to as the exploration- exploitation dilemma.'

This quote also illustrates that on the one hand decision making based on known mental models can be very efficient (like navigating based on a well-known map), but on the other hand this may prevent someone from learning even better decisions (like exploring still unknown territory). The analysis of how humans use mental models in their mental processes has led to a three-level computational architecture for contextsensitive controlled adaptation where at the base level internal simulation based on a mental model takes place, at the next level adaptation of this mental model and at the second-next level context-sensitive control over this adaptation. In this paper, it is pointed out and illustrated by examples how this three-level computational architecture can be modeled as a self-modeling network: a network in which parts of the network's own structure characteristics are represented by nodes within the same network.

\section{How Humans Use Mental Models}

In their mental processes, humans use mental models in three different ways: for internal simulation, for learning, and for (self-)control; these uses are explained as follows.

Simulation: Mental Models Simulate. Mental models are often used for internal simulation. This can take place in different forms, for example, visualisation or flashbacks (in sport or in PTSD). In his often cited book [8], Kenneth Craik describes a mental model as a small-scale model that is carried by an organism within its head:

"If the organism carries a "small-scale model" of external reality and of its own possible actions within its head, it is able to try out various alternatives, conclude which is the best of them, react to future situations ...' ([8], p. 61)

'...it is a physical working model which works in the same way as the process it parallels...' ([8], p. 51).

Internal simulation is assumed to be based on causal relations for world processes; e.g., $[9,10,11,12]$. For example, in [13], the functioning of a car in interaction with its driver is simulated based on a person's mental model, and in [14] a flashback experience of a course of events is addressed as internal simulation based on a mental model of this course of events.

Adaptation: Mental Models Adapt. Mental models are adapted often (e.g., learning, revising, forgetting); for example:

'Model-based learning is a dynamic, recursive process of learning by building mental models. It incorporates the formation, testing, and subsequent reinforcement, revision, or rejection of mental models of some phenomenon.' [15]

Observational learning concerns observation of others or of oneself while 'learning by doing' or 'learning by discovery'. Learners may see someone perform a behavior and 
then start to imitate it; e.g., [16, 17]. Mirror neurons are a basis for learning by observation; e.g., $[18,19,20]$. On the other hand, instructional learning is a useful addition as only learning based on observation may be not very efficient; e.g., [21].

Control: Mental Models Respond to Control. For learning mental models, control is exerted; see also [22, 23, 24]. Such control is also referred to as metacognition; e.g., [25, 26, 27, 28]. Also, the term self-regulation is used [28].

Overall architecture. Based on the processes for mental models summarized above, a cognitive architecture for handling mental models has been designed covering the three types of processes in an integrated manner as depicted in Figure 1.



Figure 1. A cognitive architecture for mental model handling.

Here, mental models are addressed through multiple representations: they can be viewed from three levels of representation according to the three planes depicted in Figure 1. At the base level depicted by the lower (pink) plane, a mental model, which essentially is considered to be a relational structure, is represented by its nodes and the relations between the nodes. For internal simulation, the nodes have activation levels that vary over time: based on the relations these activation levels affect each other over time. Next, at the adaptation level depicted in Figure 1 by the middle (blue) plane, it is represented how the mental model relations change over time by some adaptation specification. Finally, at the top level depicted by the upper (purple) plane in Figure 1 it is indicated how the adaptation at the middle level is controlled. For more details of this architecture and its application, see [29].

\section{Modeling by Self-Modeling Networks}

The Network-Oriented Modelling approach based on adaptive temporal-causal networks as self-modeling networks from $[30,31]$ is a suitable modeling approach to represent in an adaptive and controlled manner the causal relations and the way they can be processed to generate mental processes. Using this approach, adaptive networks of multiple orders can be modelled relatively easily. Network nodes $X$ have state values indicated by real numbers $X(t)$ that vary over time $t$; nodes are also called states. The characteristics defining a network are:

- Connectivity characteristics: connections from states $X$ to $Y$, having connection weights $\boldsymbol{\omega}_{X, Y}$ specifying their strengths

- Aggregation characteristics: each state $Y$ has a combination function $\mathbf{c}_{Y}$ that specifies how impact from all incoming connections on $Y$ is aggregated. Based on 
a list of basic combination functions $\mathbf{b c f}_{i}$ (each with some parameters) provided by an available library, such a combination function can be specified by weights $\boldsymbol{\gamma}_{i}$ and parameters $\boldsymbol{\pi}_{i, j}$ for these basic combination functions $\mathbf{b c f}_{i}$

- Timing characteristics: each state $Y$ has a speed factor $\eta_{Y}$ specifying how fast $Y$ changes

The numerical representation created by the available dedicated software environment is based on the following equations based on the above network characteristics (where $X_{1}, \ldots, X_{k}$ are the states from which state $Y$ gets incoming connections):

$$
\begin{gathered}
\operatorname{impact}_{X, Y}(t)=\omega_{X, Y} X(t) \\
\operatorname{aggimpact}_{Y}(t)=\mathbf{c}_{Y}\left(\operatorname{impact}_{X 1, Y}(t), \ldots, \operatorname{impact}_{X_{\mathrm{k}}, Y}(t)\right)=\mathbf{c}_{Y}\left(\boldsymbol{\omega}_{X_{1}, Y} X_{1}(t), \ldots, \boldsymbol{\omega}_{X_{k}, Y} X_{k}(t)\right) \\
Y(t+\Delta t)=Y(t)+\boldsymbol{\eta}_{Y}\left[\operatorname{aggimpact}_{Y}(t)-Y(t)\right] \Delta t \\
=Y(t)+\boldsymbol{\eta}_{Y}\left[\mathbf{c}_{Y}\left(\boldsymbol{\omega}_{X_{1}, Y} X_{1}(t), \ldots, \boldsymbol{\omega}_{X k, Y} X_{k}(t)\right)-Y(t)\right] \Delta t
\end{gathered}
$$

A computational network engine developed within this software environment based on the generic equations (3) takes care for the processing of all network states thereby using their connections and other network characteristics.

Self-modeling networks to model adaptivity and control. To design network models that are adaptive, the concept self-modeling network (also called reified network) introduced in $[30,31]$ has turned out to be very useful. A self-modeling network is obtained if for some of the network characteristics $\omega, \gamma, \pi, \eta$ as introduced above, network states are added to the network that represent their value. For example, for a connection weight $\boldsymbol{\omega}_{X, Y}$ an additional state $\mathbf{W}_{X, Y}$ (called a first-order self-model state) is added to the network that represents the value of this weight $\omega_{X, Y}$ and is indeed used as value for that weight in the processing. For such an additional network $\mathbf{W}$-state, also additional network characteristics are added to get an adequate embedding in the obtained self-modeling network. For example, for Hebbian learning [32] a specific combination function $\operatorname{hebb}_{\mu}\left(V_{1}, V_{1}, W\right)$ from the library can be used involving a persistence parameter $\mu$ :

$$
\operatorname{hebb}_{\mu}\left(V_{1}, V_{2}, W\right)=V_{1} V_{2}(1-W)+\boldsymbol{\mu} W
$$

Here, $V_{1}, V_{2}$ are activation levels of the connected states and $W$ is the activation level of the self-model state $\mathbf{W}_{X, Y}$ for the connection weight. As a simpler alternative, sometimes the function

$$
\operatorname{smin}_{\lambda}\left(V_{1}, V_{2}\right)=\min \left(V_{1}, V_{2}\right) / \lambda
$$

is used for Hebbian learning, where $\boldsymbol{\lambda}$ is a scaling factor; e.g., see [33].

As an example at a next (second-order self-model) level, for the combination function $\mathbf{h e b b}_{\boldsymbol{\mu}}\left(V_{1}, V_{1}, W\right)$ of a self-model state $\mathbf{W}_{X, Y}$, the persistence parameter $\boldsymbol{\mu}$ can be represented by another self-model state $\mathbf{M}_{X, Y}$. The latter network state is a second-order self-model state as it represents a network characteristic related to (first-order) self-model state $\mathbf{W}_{X, Y}$. Another example of a second-order self-model state is a state $\mathbf{H w}_{X, Y}$ that represents the speed factor (learning rate) $\eta$ of (first-order) self-model state $\mathbf{W}_{X, Y}$. These 
two types of self-model states can be used in an adaptive network model to control the learning of mental models: if the activation level of $\mathbf{M w}_{X, Y}$ gets lower, more extinction takes place which occurs when mental models are forgotten. Moreover, decreasing activation values of $\mathbf{H w}_{X, Y}$ model make the learning slow down, and a value 0 causes a complete freezing of the learning (no plasticity).

These first- and second-order self-model states directly relate to the three levels in the architecture depicted in Figure 1. Within the lowest (pink) plane the base network states are depicted, within the middle (blue) plane the first-order self-model states (such as states $\mathbf{W}_{X, Y}$ ), and within the upper (purple) plane the second-order self-model states (such as states $\mathbf{M} \mathbf{w}_{X, Y}$ and $\mathbf{H} \mathbf{w}_{X, Y}$ ).

\section{Examples of Self-Modeling Network Models for Mental Models}

In this section, as illustrations two examples of self-modeling network models for mental models are briefly discussed. They are both instantiations of the generic three-level architecture discussed in Section 2.

Using metaphors as mental models. This example (described in more detail in [34]) addresses the use of metaphors as mental models to support joint decision making; see Figure 2 for a part of this network model. At the base levels two different metaphor options are modeled: cooperative metaphor state met $_{c o o}$ and competitive metaphor state $\mathrm{met}_{\text {com }}$; see the lower (pink) plane in Figure 2. The incoming and outgoing connections for these metaphor states are adaptive, modeled by the first-order self-model $\mathbf{W}$-states; see the middle (blue) plane. The control of the adaptation of all these $\mathbf{W}$-states is modeled by the second-order self-model $\mathbf{H w}_{w}$-state; see the upper (purple) plane.



Figure 2 Connectivity of a part of an adaptive network model for mental models for metaphors with their activation and effect connections at the base level, their learning at the first-order self-model level and the control of the learning at the second-order self-model level.

The two $\mathbf{W}$-states for the incoming connections for the metaphor states determine when the metaphor should be activated. These connections are learnt by Hebbian 
learning, which has as effect that the more often they are used in a certain context (represented by stimulus representation $\mathrm{srs}_{s}$ ), the stronger their activations become when that context occurs. The four $\mathbf{W}$-states for the outgoing connections from the metaphor states determine the effect of the metaphor states on the self-owner states; they are adapted based on whether the other person tends to go for action $a c$, and whether the other person has a good feeling bo about it (states $\operatorname{srs}_{B, a c}$ and $\operatorname{srs}_{B, b o}$ ). For each of these six $\mathbf{W}$-states, its adaptation is controlled through the second-order self-model $\mathbf{H w}$-state which represents the learning rate of the related $\mathbf{W}$-state. This form of second-order adaptation is based on the second-order adaptation principle 'adaptation accelerates with increasing stimulus exposure' [4].

Learner-controlled learning. The second example (described in more detail in [35]) addresses how a learner controls at what times instruction by a teacher takes place; see Figure 3 for a representative part of this model. Here the three $\mathbf{R W}$-states in the middle (blue) plane represent the weights of the connections in the mental model (that is learned) depicted at the (pink) base level plane. For example, state $\mathbf{R} \mathbf{W}_{\text {Switch,TurnSwitch }}$ within the first-order self-model plane, also indicated by $\mathrm{X}_{31}$, represents the weight of the connection in the base plane from state $\mathrm{BS}_{\text {Switch }}$ to state $\mathrm{BS}_{\text {TurnSwitch }}$ (the arrow from $\mathrm{X}_{1}$ to $\left.\mathrm{X}_{2}\right)$.

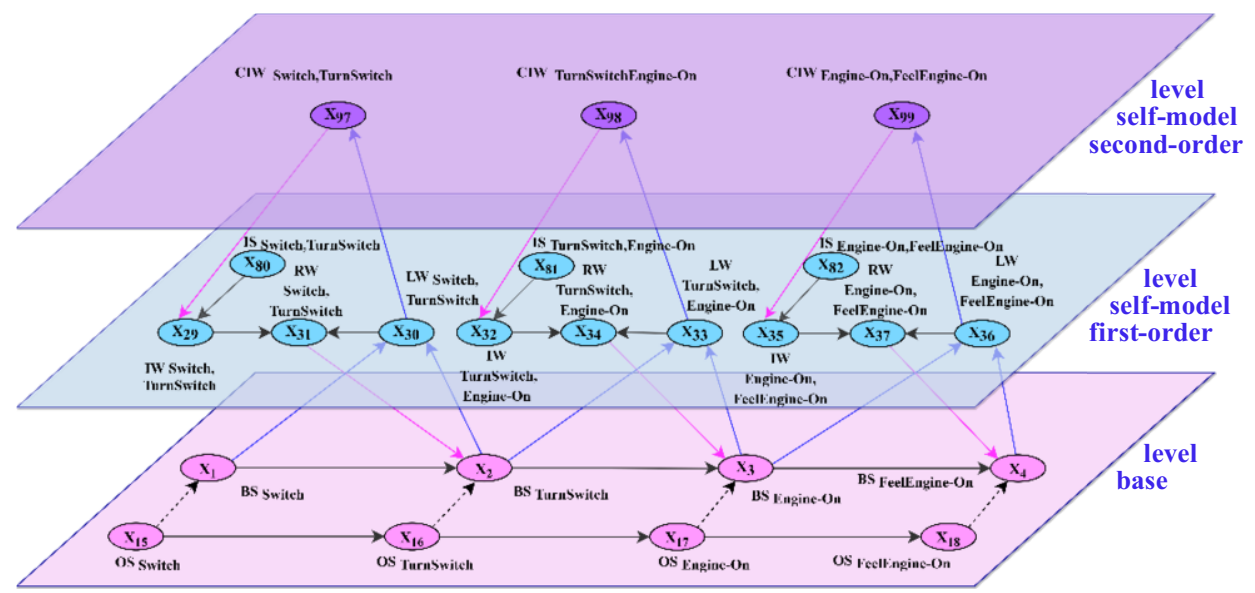

Figure 3. Connectivity of a part of an adaptive network model for mental models for learner control of a teacher's instruction.

These $\mathbf{R W}$-states are based on input from observational learning (modeled by the corresponding $\mathbf{L W}$-states) and instructional learning (modeled by the corresponding IWstates). For example, state $\mathbf{R W}_{\text {Switch,TurnSwitch }}$ has incoming connections from

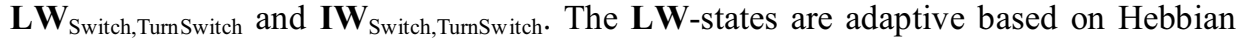
learning; to this end they use combination function (4) from Section 3. For this scenario, they are not (explicitly) controlled. In contrast, for the IW-states, their incoming connections from the IS-states (representing the communication channel from the information source IS-state, which is the teacher) are controlled. This control is modeled by the second-order self-model CTW-states at the upper (purple) level; these are also denoted as $\mathbf{W}_{\mathbf{W}}$-states. For example, state $\mathbf{C} \mathbf{I} \mathbf{W}_{\text {Switch, TurnSwitch }}$ in the (purple) second-order self-model plane controls the connection from $\mathbf{I S}_{\text {Switch,TurnSwitch }}$ to $\mathbf{I W}_{\text {Switch,TurnSwitch, which }}$ 
models the communication from instructor to learner about the connection from $\mathrm{BS}_{\mathrm{Switch}}$

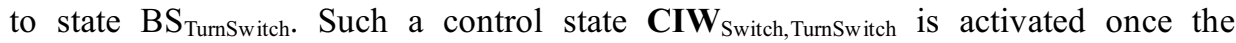
corresponding $\mathbf{L W}$-state $\mathbf{L W}_{\text {Switch,TurnSwitch }}$ has a sufficient action level: the learner has (partially) learnt it based on observation and asks the teacher for confirmation. So, these CIW-states determine when the communication channels have to be opened for the instructional learning.

Learning by counterfactual thinking. A third case to illustrate the approach is adopted from [36]; it addresses learning by counterfactual thinking; see Figure 4. Here after a negatively evaluated experience modelled by the red box in the base plane, the person considers internal simulation of mental models for alternative action options and their expected results (see the orange box in the base plane). The counterfactual status of this is modelled by the process modelled in the middle blue plane and that process is controlled by the upper level CS-states in the purple plane. See [36] for more details.

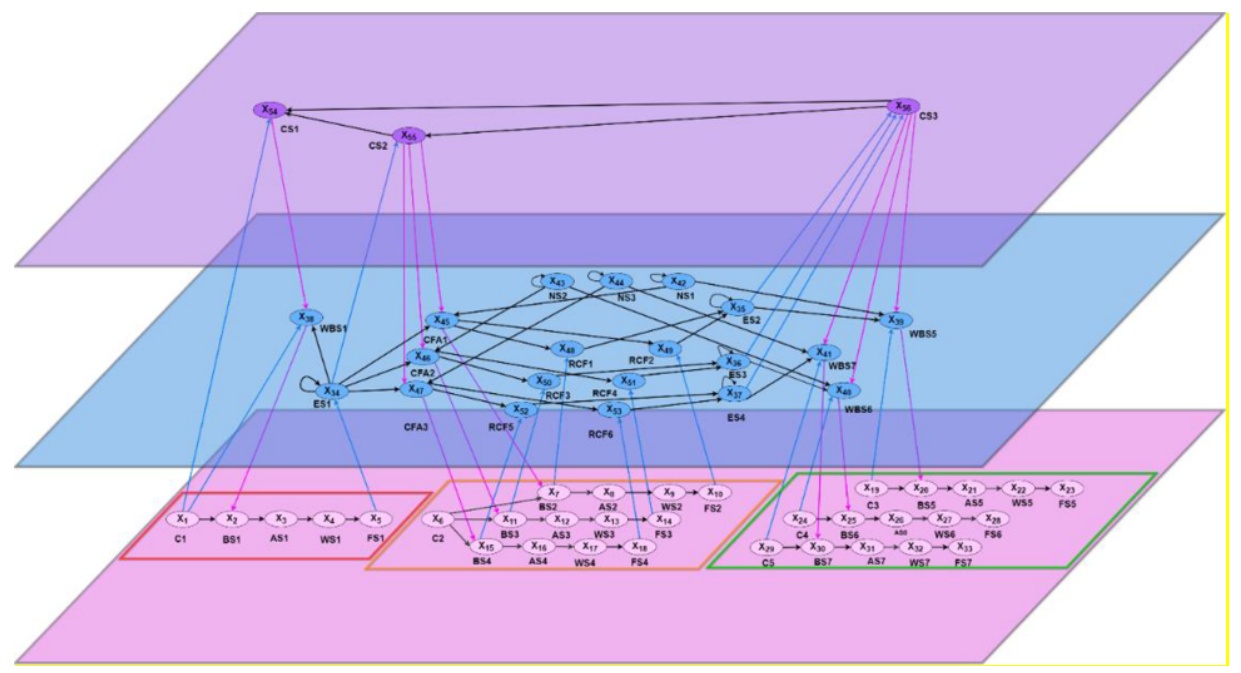

Figure 4. Connectivity of the controlled adaptive network model for counterfactual thinking.

\section{Options for Control of Adaptation using Self-Modelling Networks}

In Section 4, some examples of adaptation control for mental models were discussed, for two cases within the context of self-modelling networks used as a vehicle. In this section, a more systematic overview is discussed of different ways in which control over mental models and their adaptation can be exerted, thereby again making use of the network structure provided by self-modelling networks. In a controlled adaptive network model for mental models based on a self-modelling network, adaptation is modelled by a firstorder self-model. There are a number of network characteristics involved in the firstorder self-model states used for the adaptation. In particular, by systematically going through all possible network characteristics, the following examples of network characteristics for adaptation to be controlled can be distinguished and are illustrated by various examples: 


\section{- Control by adaptive connectivity characteristics of first-order self-model states}

- Adaptive connections of the causal pathways to the self-model states and their weights $\omega$; for example:

- Choosing a mental model to be applied. For example, a decision to use a specific metaphor-based mental model as in the model in Section 4 taken from [34] or a decision to use a geometric mental model to support learning of an arithmetic mental model, as described in [37]

- Opening a communication channel from an information source to enable instructional learning of a mental model (decision to ask), as in the model in Section 4 taken from [35] and in the model described in [38]

- Opening an observation channel to enable observational learning of a mental model (decision to observe), as in the model described in [38]

- Adaptive connections of the causal pathways from the self-model states to other states and their weights $\omega$; for example:

- Modelling the effects of a chosen metaphor as in the model in Section 4 taken from [34]

- Control by adaptive aggregation characteristics of first-order self-model states

- Adaptive choice of combination function; for example:

- For Hebbian learning of mental model connections a weighted average of $\operatorname{hebb}_{\mu}\left(V_{1}, V_{2}, W\right)$ and $\operatorname{smin}_{\lambda}\left(V_{1}, V_{2}\right)$, with adaptive weights $\gamma_{1}$ and $\gamma_{2}$.

- Adaptive parameters of chosen combination functions; for example:

- Adaptive values for the persistence factor $\boldsymbol{\mu}$ of $\mathbf{h e b}_{\mu}(.$.$) as in the self-modelling network model$ for shared mental models described in [38] or for the scaling factor $\lambda$ of $\operatorname{smin}_{\lambda}\left(V_{1}, V_{2}\right)$.

\section{- Control by adaptive timing characteristics of first-order self-model states}

$\circ$ Adaptive adaptation speed (learning rate) $\eta$; for example:

- Addressing the Plasticity Versus Stability conundrum [1] based on some context factors indicating when plasticity is needed fully and when plasticity should be limited or frozen.

- Accelerating adaptation speed upon increased stimulus exposure [4], for example as applied in the example model in Section 3 taken from [34]

In Table 1 an overview is given of a large number of applications of such self-models to model human processes based on mental models as brought together in book [29].

Recall from Section 3 how at some level self-model states can be introduced to represent network characteristics from a lower level. The naming is as follows: selfmodel state $\mathbf{W}_{X, Y}$ represents connection weight $\boldsymbol{\omega}_{X, Y}$ from the lower level, $\mathbf{H}_{Y}$ represents speed factor $\eta_{Y}$ from the lower level, and so on. Such self-model states can be generally called $\mathbf{W}$-states or $\mathbf{H}$-states, for example. By iterating this, for example, second-order self-model state $\mathbf{H w}_{X, Y}$ represents the adaptation speed of first-order self-model state $\mathbf{W}_{X, Y}$ and second-order self-model state $\mathbf{M w}_{X, Y}$ represents the persistence parameter $\boldsymbol{\mu w}_{X, Y}$ of first-order self-model state $\mathbf{W}_{X, Y}$. Similarly, second-order self-model state $\mathbf{W}_{Z, \mathbf{W}_{X, Y}}$ represents the weight $\omega Z, \mathbf{W}_{X, Y}$ of the connection from some state $Z$ to state $\mathbf{W}_{X, Y}$. Such second-order self-model states can be generally called Hw-states, Mw-states, or Wwstates. Note that in most cells in Table 1 further references are included, but for those cells where no references are included, in general this means that these options are yet to be addressed in detail. 
Table 1 Overview of different types of controlled adaptation for example mental models as collected in [29]

\begin{tabular}{|c|c|c|c|}
\hline Learning by & $\begin{array}{l}\text { Control of adaptation } \\
\text { via connectivity }\end{array}$ & $\begin{array}{l}\text { Control of adaptation } \\
\text { via aggregation }\end{array}$ & $\begin{array}{l}\text { Control of adaptation } \\
\text { via timing }\end{array}$ \\
\hline $\begin{array}{l}\text { Observation and } \\
\text { monitoring }\end{array}$ & $\begin{array}{l}\text { Controlled learning by } \\
\text { observation for a mental model } \\
\mathbf{W} \text {-state for bonding via a } \mathbf{W}_{\mathbf{W}^{-}} \\
\text {state ([29], Ch 13; formation of a } \\
\text { mental model of another person; } \\
\text { see also [33]) }\end{array}$ & $\begin{array}{l}\text { Hebbian mental model } \\
\text { learning } \mathbf{W} \text {-state persistence } \\
\text { control via an } \mathbf{M}_{\mathbf{W}} \text {-state } \\
\text { ([29], Ch 14; controlled } \\
\text { forgetting of a mental model } \\
\text { relation; see also [39]) }\end{array}$ & $\begin{array}{l}\text { Hebbian mental model learning } \mathbf{W} \text { - } \\
\text { state speed control via an } \mathbf{H}_{\mathbf{W}} \text {-state: } \\
\text { adaptation accelerates with } \\
\text { increasing exposure ([29], Ch } 5, \mathrm{Ch} \\
7, \mathrm{Ch} 10, \mathrm{Ch} 11, \mathrm{Ch} 12 \text {; e.g., } \\
\text { learning mental models for flashback } \\
\text { experiences [14], analysis and } \\
\text { support tasks [40], metaphors [34], } \\
\text { God-models [41], self- and other- } \\
\text { models [33]) }\end{array}$ \\
\hline $\begin{array}{l}\text { Excitability } \\
\text { adaptation }\end{array}$ & $\begin{array}{l}\text { Incoming connection for an } \\
\text { adaptive mental model } \\
\text { excitability T-state control via a } \\
\mathbf{W}_{\mathbf{T}} \text {-state ([29], Ch 7; learning } \\
\text { excitability [42, 43] of a mental } \\
\text { model's states; see also [40]) }\end{array}$ & $\begin{array}{l}\text { Excitability mental model } \\
\text { learning } \mathbf{T} \text {-state aggregation } \\
\text { control, for example through } \\
\text { adaptive (steepness } \boldsymbol{\sigma} \text { and } \\
\text { threshold } \boldsymbol{\tau} \text { ) parameters of a } \\
\text { logistic combination } \\
\text { function used for the } \mathbf{T} \text {-state } \\
\text { represented by } \mathbf{S}_{\mathbf{T}^{-}} \text {and } \mathbf{T}_{\mathbf{T}^{-}} \\
\text {states }\end{array}$ & $\begin{array}{l}\text { Excitability mental model learning } \\
\text { T-state speed control via an } \mathbf{H}_{\mathbf{T}} \text {-state } \\
\text { ([29], Ch 7; learning excitability [42, } \\
\text { 43] of a mental model's states; see } \\
\text { also [40]) }\end{array}$ \\
\hline Communication & $\begin{array}{l}\text { Learner-controlled instructional } \\
\text { learning of a mental model } \mathbf{W} \text { - } \\
\text { state via a } \mathbf{W}_{\mathbf{W}} \text {-state ([29], Ch } 9 \text {; } \\
\text { opening a communication } \\
\text { channel with the instructor by } \\
\text { asking; see also [35]) } \\
\text { Controlled learning by } \\
\text { communication for a mental } \\
\text { model W-state for bonding via a } \\
\mathbf{W}_{\mathbf{W}} \text {-state ([29], Ch } 13 \text { opening a } \\
\text { communication channel with the } \\
\text { other person by asking; see also } \\
\text { [38]) }\end{array}$ & $\begin{array}{l}\text { Learner-controlled } \\
\text { instructional learning of a } \\
\text { mental model } \mathbf{W} \text {-state via a } \\
\mathbf{T}_{\mathbf{W}} \text {-state for excitability [ } 42 \text {, } \\
43 \text { ] of the } \mathbf{W} \text {-state (opening } \\
\text { a communication channel } \\
\text { with the instructor by more } \\
\text { sensitive listening) }\end{array}$ & $\begin{array}{l}\text { Learner-controlled instructional } \\
\text { learning of a mental model } \mathbf{W} \text {-state } \\
\text { via an } \mathbf{H}_{\mathbf{W}} \text {-state (controlling the } \\
\text { timing of a communication channel } \\
\text { with the instructor) }\end{array}$ \\
\hline $\begin{array}{l}\text { Contextual } \\
\text { factors }\end{array}$ & $\begin{array}{l}\text { Controlled connection } \mathbf{W} \text {-state } \\
\text { for activation of mental model via } \\
\text { a } \mathbf{W}_{\mathbf{W}} \text {-state ([29], Ch } 6 \text {; activation } \\
\text { of a mental model for possible } \\
\text { future action; see also [36]) }\end{array}$ & $\begin{array}{l}\text { Controlled connection } \mathbf{W} \text { - } \\
\text { state for activation of mental } \\
\text { model via a } \mathbf{T}_{\mathbf{W}} \text {-state } \\
\text { addressing excitability of } \\
\text { the } \mathbf{W} \text {-state based on } \\
\text { contextual factors }\end{array}$ & $\begin{array}{l}\text { Controlled adaptive mental model } \\
\text { effect connection } \mathbf{W} \text {-state via an } \\
\mathbf{H}_{\mathbf{W}} \text {-state ([29], Ch 10; adapting the } \\
\text { own choices based on the context } \\
\text { given by the other person; see also } \\
[34])\end{array}$ \\
\hline
\end{tabular}

\section{Discussion}

This paper addressed how in mental and social processes, humans often apply specific mental models and learn and adapt them in a controlled manner. It was explored how analysis of such processes may provide useful inspiration for the development of computational approaches from an AI, computational intelligence and machine learning 
perspective. It was discussed how controlled adaptation relates to the Plasticity Versus Stability Conundrum in neuroscience [1]. From the analysis a three-level computational architecture for controlled adaptation was obtained. It was discussed how this three-level computational architecture can be modeled as a self-modeling network [30, 31], illustrated by a number of examples.

The work discussed here can be considered as part of the area of human-like machine learning. Also, within machine learning in general there are a few approaches that do (explicitly) address control of adaptation. A simple example is Simulated Annealing, where the temperature parameter can be considered an adaptive adaptation control parameter. Another example is the Explore-Exploit Dilemma in reinforcement learning, where the challenge is to find criteria on the context that can be used to decide in a context-sensitive manner between explore or exploit; e.g., $[5,6,7]$. Two limitations of the current state of the presented work that may be identified are:

- The approach has only been explored yet for deterministic models. In principle, probabilistic models are possible when combination functions are added to the library with some probabilistic or random effects, but this still has to be explored in more detail

- Analysis of the wider domain of human-like approaches to control of adaptation, in particular for mental model adaptation as discussed here, may provide inspiration for further development of machine learning methods. This is also an area that has not yet been explored in more detail.

It may be noted from this paper that for human-like controlled adaptation much input and inspiration was obtained from the area of neuroscience; for example, in [1, 2, 3, 4] and many other papers on metaplasticity several second-order adaptation principles for context-sensitive control of adaptation can be found. This is in strong contrast with the area of social science. Literature on adaptive social relations or adaptive social networks is available including computational models for it, such as [44, 45], but although adaptation control is certainly also in this area a very relevant factor, approaches addressing the control of adaptation seems to be lacking in such literature, where only just a few more or less incidental exceptions can be found such as $[6,30,34,38,39,46$, 47] and [31], Ch 6. Much progress would become possible for social science by explicitly addressing this issue more systematically and on a much larger scale like it was already done within neuroscience.

\section{References}

[1] Sjöström PJ, Rancz EA, Roth A, Hausser M. Dendritic excitability and synaptic plasticity. Physiol Rev 2008; 88: 769-840.

[2] Abraham WC, Bear MF. Metaplasticity: the plasticity of synaptic plasticity. Trends in Neuroscience 1996; 19(4):126-130.

[3] Magerl W, Hansen N, Treede RD, Klein T. The human pain system exhibits higher-order plasticity (metaplasticity). Neurobiology of Learning and Memory 2018; 154:112-20.

[4] Robinson BL, Harper NS, McAlpine D. Meta-adaptation in the auditory midbrain under cortical influence. Nat. Commun. 2016; 7: article 13442.

[5] Holland JH. Adaptation in natural and artificial systems. Ann Arbor, MI: University of Michigan Press; 1975.

[6] March JG. Exploration and exploitation in organizational learning. Organization Science 1991; 2: 71-87.

[7] Wilson RC, Geana A, White JM, Ludvig E, Cohen JD. Humans use directed and random exploration to solve the explore-exploit dilemma. J. Experimental Psychol: General 2014; 143(6): 2074-2081.

[8] Craik KJW. The nature of explanation. Cambridge, MA: University Press; 1943. 
[9] Damasio AR. Descartes error: emotion, reason and the human brain. London: Vintage Books; 1994.

[10] Goldman AI. Simulating minds: the philosophy, psychology, and neuroscience of mindreading, Oxford University Press, New York; 2006.

[11] Hesslow G. Conscious thought as simulation of behaviour and perception, Trends Cogn. Sci. 2002; 6(2002), 242-247.

[12] Hesslow G. The current status of the simulation theory of cognition. Brain Res. 2012; 1428: 71-79.

[13] Bhalwankar R, Treur J. Modeling the development of internal mental models by an adaptive network model. In Proc. of the 11th Annual International Conference on Brain-Inspired Cognitive Architectures for AI, BICA*AI'20. Procedia Computer Science, Elsevier Publishers; 2021.

[14] Van Ments L, Treur J. A higher-order adaptive network model to simulate development of and recovery from PTSD. In: Paszynski M, Kranzlmüller D, Krzhizhanovskaya VV, Dongarra JJ, Sloot PMA (eds) Computational Science - Proc. ICCS 2021. Lecture Notes in Computer Science, vol 12743. Springer Nature, Cham; 2021. p. 154-166.

[15] Buckley BC. Interactive multimedia and model-based learning in biology. Intern. J. Sci. Education 2000; 22(9): 895-935.

[16] Benbassat J. Role modeling in medical education: the importance of a reflective imitation. Academic Medicine 89(4); 550-554: 2014.

[17] Yi MY, Davis FD. Developing and validating an observational learning model of computer software training and skill acquisition. Information Syst. Res. 14(2); 146-169: 2003.

[18] Hurley S. The shared circuits model (SCM): How control, mirroring, and simulation can enable imitation, deliberation, and mindreading. Behavioral Brain Sci. 2008; 31(1), 1-22.

[19] Rizzolatti G, Craighero L. The mirror-neuron system. Annu. Rev. Neurosci. 2004; 27: 169-192.

[20] Van Gog T, Paas F, Marcus N, Ayres P, Sweller J. The mirror neuron system and observational learning: Implications for the effectiveness of dynamic visualizations. Educ. Psychol. Rev. 2009; 21(1): 21-30.

[21] Seel NM. Mental models in learning situations. In: Advances in Psychology, vol. 138. Amsterdam: North-Holland; 2006. p. 85-107.

[22] Gibbons J, Gray M. an integrated and experience- based approach to social work education: The Newcastle model. Social Work Education 2002; 21: 529-549.

[23] Hogan KE, Pressley ME. Scaffolding student learning: Instructional approaches and issues. Brookline Books; 1997.

[24] Kozma RB. Learning with media. Review of educational research 1991; 61(2): 179-211.

[25] Darling-Hammond L, Austin K, Cheung M, Martin D. Thinking about thinking: Metacognition. In The Learning Classroom: Theory into Practice. Stanford University School of Education 2008. p. 157-172.

[26] Mahdavi M. An overview: metacognition in education. Intern. J. Multidisciplinary Current Res. 2014; $2: 529-535$.

[27] Koriat A. Metacognition and consciousness. In: Zelavo PD, Moscovitch M, Thompson E (eds.). Cambridge Handbook of Consciousness. New York: Cambridge University Press. 2007.

[28] Pintrich PR. The role of goal orientation in self-regulated learning. In: Boekaerts M, Pintrich P, Zeidner M (eds.). Handbook of self-regulation research and applications. Orlando, FL: Academic Press. 2000, p. 451-502.

[29] Treur J, Van Ments L (eds.). Mental Models and their Dynamics, Adaptation and Control: A Selfmodeling Network Modeling Approach. Springer Nature, Cham. 2022.

[30] Treur J. Modeling higher-order adaptivity of a network by multilevel network reification. Network Science 2020; 8: S110-44.

[31] Treur J. Network-oriented modeling for adaptive networks: designing higher-order adaptive biological, mental, and social network models. Cham, Switzerland: Springer Nature Publishing; 2020. 412 p.

[32] Hebb DO. The organization of behavior: A neuropsychological theory. New York: John Wiley and Sons; 1949. $335 \mathrm{p}$.

[33] Hermans A, Muhammed S, Treur J. An Adaptive network model for attachment theory. In: Paszynski M, Kranzlmüller D, Krzhizhanovskaya VV, Dongarra JJ, Sloot PMA (eds) Computational Science - Proc. ICCS 2021. Lecture Notes Comp. Sci., vol 12744. Springer Nature, Cham. 2021. p. 462-475.

[34] Van Ments L, Treur J. Modeling adaptive cooperative and competitive metaphors as mental models for joint decision making. Cognitive Systems Research 2021; 69: 67-82. https://doi.org/10.1016/j.cogsys.2021.06.002.

[35] Bhalwankar R, Treur J. A second-order adaptive network model for learner-controlled mental model learning processes. In: Proc. of the 9th International Conference on Complex Networks and their Applications, vol. 2. Studies in Computational Intelligence, vol. 944. Springer Nature Switzerland AG; 2021. p. 245-259. Extended version to appear in PLOS One.

[36] Bhalwankar R, Treur J. 'If Only I Would Have Done that...': A Controlled Adaptive Network Model for Learning by Counterfactual Thinking. In: Proceedings of the 17th International Conference on Artificial 
Intelligence Applications and Innovations, AIAI'21 Advances in Information and Communication Technology, vol. 627. Springer Nature; 2021. p. 3-16.

[37] Treur J. An adaptive network model covering metacognition to control adaptation for multiple mental models. Cognitive Syst. Res. 2021; 67: 18-27.

[38] Treur J. Modeling controlled social network adaptation using mental models. Proc. of the $7^{\text {th }}$ International Congress on Information and Communication Technology, ICICT'21. Advances in Intelligent Systems and Computing, Springer Nature, in press. 2021.

[39] Van Ments L, Treur J, Klein J, Roelofsma PHMP. A second-order adaptive network model for shared mental models in hospital teamwork. In: Nguyen NT, et al. (eds.), Proc. of the 13th International Conference on Computational Collective Intelligence, ICCCI'21. Lecture Notes in AI, Springer Nature; 2021

[40] Treur J. Self-modeling networks using adaptive internal mental models for cognitive analysis and support processes. In: Proc. of the 9th International Conference on Complex Networks and their Applications, vol. 2. Studies in Computational Intelligence, vol. 944. Springer Nature Switzerland AG. 2021, p. 260274. Extended version: A Self-Modeling Network Model Addressing Controlled Adaptive Mental Models for Analysis and Support Processes. Complex Systems, 2021, in press.

[41] Van Ments L, Treur J, Roelofsma PHMP. How empathic is your god: an adaptive network model for formation and use of a mental god-model and its effect on human empathy. In: Treur J, Van Ments L (eds.), Mental Models and their Dynamics, Adaptation and Control: A Self-modeling Network Modeling Approach. Springer Nature, Cham; 2022.

[42] Debanne D, Inglebert Y, Russier M. Plasticity of intrinsic neuronal excitability. Current Opinion in Neurobiology 2019; 54: 73-82.

[43] Chandra N, Barkai E. A non-synaptic mechanism of complex learning: modulation of intrinsic neuronal excitability. Neurobiology of Learning and Memory 2018; 154: 30-36.

[44] McPherson M, Smith-Lovin L, Cook JM. Birds of a feather: homophily in social networks. Annu. Rev. Sociol. 2001; 27: 415-44.

[45] Treur J. Mathematical analysis of the emergence of communities based on coevolution of social contagion and bonding by homophily. Appl. Network Sci. 2019; 4: article 1.

[46] Carley KM. Inhibiting Adaptation. In: Proceedings of the 2002 Command and Control Research and Technology Symposium. Monterey, CA: Naval Postgraduate School; 2002. p. 1-10.

[47] Carley KM. Destabilization of covert networks. Comput. Math Organiz. Theor. 2006; 12: 51-66. 